\title{
Nuclear modifications of fragmentation functions and rescaling models * $\dagger$
}

\author{
Alberto Accardi a $\ddagger$ and Hans J. Pirner ${ }^{a b \S}$ \\ anstitut für Theoretische Physik der Universität Heidelberg, Germany

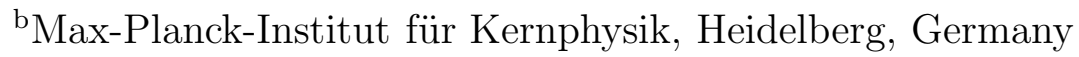

We discuss nuclear modification of fragmentation functions in the context of the socalled "rescaling models". These models implement partial deconfinement inside nuclei by modifying the fragmentation functions perturbatively. We apply these models to the analysis of nuclear hadron production in deep inelastic scattering processes at the HERMES and EMC experiments.

In deep inelastic scattering a projectile lepton $\ell$ emits a virtual photon $\gamma^{*}$, which scatters on a quark $q$ from the target $T$, in our case a deuteron $D$ or a heavier nucleus with atomic number $A$. The struck quark fragments into the observed hadron $h$, see Fig. 1. We use kinematic variables as summarized in the same figure: $x$ is the Bjorken's scaling variable, $\nu$ is the energy of the virtual photon in the target rest frame, and $z$ is the fraction of the energy transferred to the produced hadron.

The experimental data on nuclear effects in hadron production are usually presented in terms of the multiplicity ratio

$$
R_{M}^{h}(z)=\frac{1}{N_{A}^{\ell}} \frac{d N_{A}^{h}}{d z} / \frac{1}{N_{D}^{\ell}} \frac{d N_{D}^{h}}{d z}
$$

where $N_{A}^{\ell}$ is the number of outgoing leptons in DIS processes on a nuclear target of atomic number $A$ and $d N_{A}^{h} / d z$ is the z-distribution of produced hadrons in the same processes; the subscript $D$ refers to the same quantities when the target is a deuteron. A similar definition for the multiplicity ratio as a function of $\nu$ may be written. In absence of nuclear effects the ratio $R_{M}^{h}$ would be equal to 1 . The experimental observation that $R_{M}^{h} \neq 1$ [1] 3 ] has been explained theoretically in many ways: as an effect of nuclear absorption of the produced hadrons [4,5], in a gluon-bremsstrahlung model for leading hadron production [6], as an higher-twist effect [7]. In this short note we will not discuss these models in detail (see Ref. [8]) and, instead, will concentrate on the so-called rescaling models [9 11].

*Talk presented at nthe "European workshop on the QCD structure of the nucleon" (QCD-N'02), Ferrara (ITA), April 3rd-6th, 2002.

${ }^{\dagger}$ This work is (partially) funded by the European Commission IHP program under contract HPRN-CT2000-00130.

${ }^{\ddagger}$ E-mail address: accardi@tphys.uni-heidelberg.de

${ }^{\S}$ E-mail address: pir@tphys.uni-heidelberg.de 


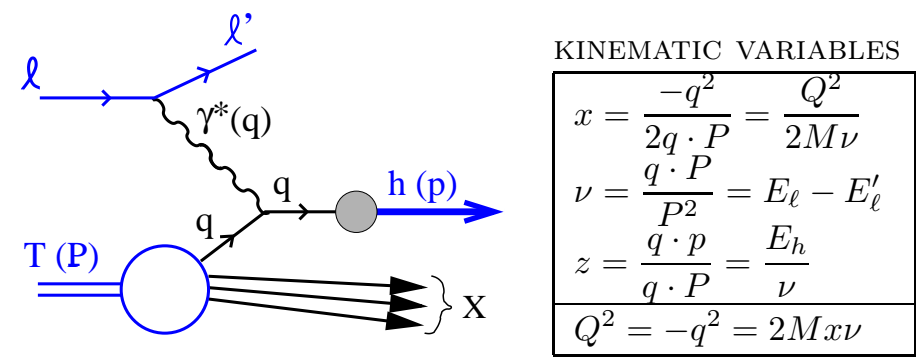

Figure 1. Kinematic variables for DIS scattering; four-moments are indicated in parentheses.

The starting point of rescaling models is to assume a change in the confinement scale $\lambda_{A}$ in nuclei, compared to the confinement scale $\lambda_{0}$ in free nucleons:

$$
\lambda_{A}>\lambda_{0}
$$

This assumed partial deconfinement in nuclei affects both the parton distribution functions (PDF) and the fragmentation functions (FF). For what concerns PDF's [9], 10, consider a valence quark which carries a momentum $Q_{0}$ when it is confined on a scale $\lambda_{0}$. If the scale changes to $\lambda_{A}$ it carries a corresponding momentum $Q_{A}$. Since there is no other dimensionful scale, the product $Q \lambda$ must remain constant, so that

$$
Q_{0} \lambda_{0}=Q_{A} \lambda_{A}
$$

Therefore, if we take $Q_{0}$ to be the initial scale for the DGLAP evolution of distribution functions, we may set

$$
q_{f}^{A}\left(x, Q_{A}=\frac{\lambda_{0}}{\lambda_{A}} Q_{0}\right)=q_{f}\left(x, Q_{0}\right)
$$

where $q_{f}$ is the distribution function of a quark of flavour $f$ in a free nucleon and $q_{f}^{A}$ is the same quantity when the nucleon is inside a nucleus. For FF's [11] a similar argument holds because constituent quark and effective hadron masses are sensitive to the confinement scale. Therefore if we take $Q_{0}$ to be the physical threshold for hadron production we may set

$$
D_{f}^{h \mid A}\left(x, Q_{A}=\frac{\lambda_{0}}{\lambda_{A}} Q_{0}\right)=D_{f}^{h}\left(x, Q_{0}\right)
$$

where $D_{f}^{h}$ is the fragmentation function of a quark of flavour $f$ into a hadron $h$ and $D_{f}^{h \mid A}$ is the nuclear modified fragmentation function. To extend Eqs. (3) and (4) to an arbitrary scale $Q$ we apply pQCD evolution. The nuclear structure and fragmentation functions evolve over larger interval in momentum compared to the corresponding functions at the same scale Q, since the starting scale is smaller, see Eq. (2). The final result [9 11] is

$$
\begin{aligned}
q_{f}^{A}(x, Q) & =q_{f}\left(x, \xi_{A}(Q) Q\right) \\
D_{f}^{h \mid A}(z, Q) & =D_{f}^{h}\left(x, \xi_{A}(Q) Q\right),
\end{aligned}
$$

where the scale factor $\xi_{A}(Q)$ is greater than one. These two equations are the main tool which we will exploit. There are two models for the computation of the scale factor: 

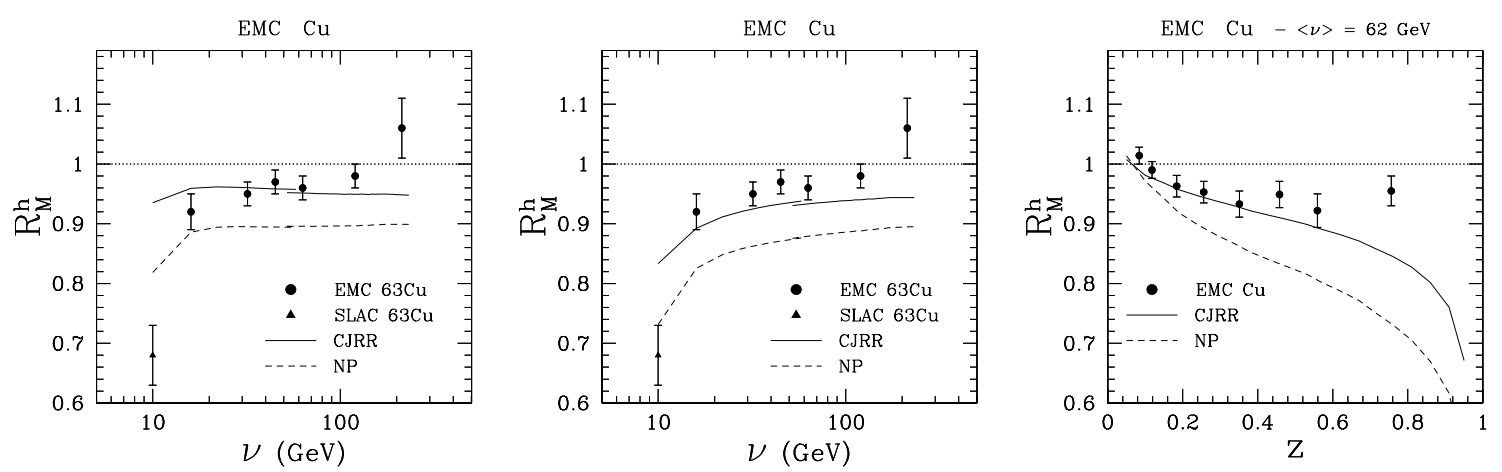

Figure 2. Comparison of partial and maximal dconfinement models at EMC with a copper target. a) $\nu$-distribution with rescaling only and b) with rescaling and absorption; c) $z$-distribution with rescaling and absorption. Data taken from Ref. [2].

a) the maximal deconfinement model (NP) [9] assumes the onset of "colour conductivity" in nuclei and takes $\lambda_{A}=R_{A}$, where $R_{A}$ is the nuclear radius, so that

$$
\xi_{A}(Q)=R_{A} / R_{p} ;
$$

b) the partial deconfinement model (CJRR) [10] assumes the deconfinement scale $\lambda_{A}$ to be proportional to the degree of overlap of the nucleons inside the given nucleus, and

$$
\xi_{A}(Q)=\left(\lambda_{A} / \lambda_{0}\right)^{\frac{1}{2} \frac{\alpha_{s}\left(Q_{0}\right)}{\alpha_{s}(Q)}} .
$$

Note that the maximal deconfinement model assumes a much larger scale factor, which results in a larger nuclear modification of hadron production at high $z$.

We calculate the multiplicity ratio (11) using the rescaled $\mathrm{PDF}$ (5) and the rescaled FF (6) in the leading order pQCD computation of $N_{A}^{\ell}$ and $d N_{A}^{h} / d z$ :

$$
\begin{aligned}
\frac{1}{N_{A}^{\ell}} \frac{d N_{A}^{h}}{d z} & =\frac{1}{\sigma^{*} A} \int_{\text {exp. cuts }} d x d \nu \sum_{f} e_{f}^{2} q_{f}\left(x, \xi_{A} Q\right) \frac{d \sigma^{\gamma^{*} q}}{d x d \nu} D_{f}^{h}\left(z, \xi_{A} Q\right) \\
\sigma^{\gamma^{*} A} & =\int_{\text {exp. cuts }} d x d \nu \sum_{f} e_{f}^{2} q_{f}\left(x, \xi_{A} Q\right) \frac{d \sigma^{\gamma^{*} q}}{d x d \nu},
\end{aligned}
$$

where $e_{f}$ is the electric charge of a quark of flavour $f, d \sigma^{\gamma^{*} q} / d x d \nu$ is the differential crosssection for a $\gamma^{*} q$ scattering computed in pQCD at leading order, and $Q^{2}=2 M x \nu$, with $M$ the nucleon mass. In the numerical computations we used GRV98 parton distribution funtions [12] and Kretzer's parametrization of FF's at leading order [13].

The results for the $\nu$-dependence of the multiplicity ratio for charged hadrons are shown in Fig. 2 2 a and compared to EMC data [2]. The maximal deconfinement model underestimates the data over nearly the whole range. On the contrary the partial deconfinement model fit the data at high $\nu$, but overestimates them at smaller $\nu$. Because of Lorentz dilatation, at high $\nu$ the hadron is formed mainly outside the nucleus and it is affected by rescaling effects only. On the contrary, at small $\nu$ the hadron is formed inside the nucleus and starts interacting with its nucleons, with some probability of being absorbed.

To take into account nuclear absorption, we follow the analysis of Ref. [4, 河: the quark propagates in the nucleus with a cross-section $\sigma_{q}$ for inelastic scattering on the nucleons. 

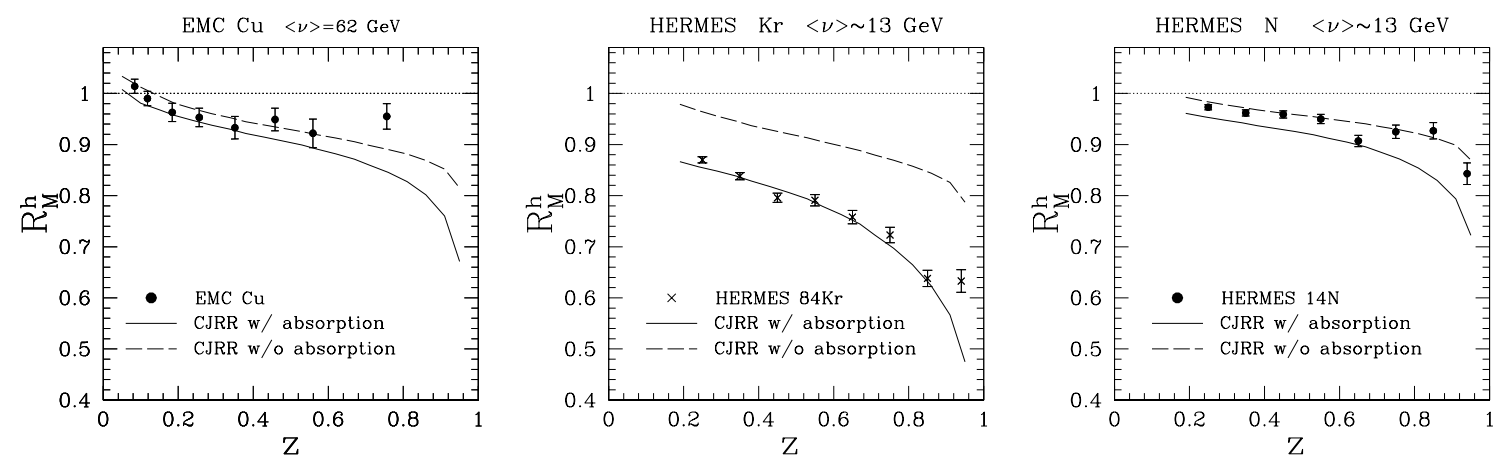

Figure 3. Comparison of absorption effects in $z$-distributions with rescaling only (dashed lines) and with rescaling and absorption (solid lines). From left to right: EMC with copper target, HERMES with krypton and HERMES with nitrogen targets. Data taken from Refs. [2,3]. Note that the scale in Fig. 3a is different from Fig. 2ac.

Subsequently, it creates a pre-hadronic states, which has a cross-section $\sigma_{*}$, and finally the observed hadron is formed, which has a cross-section $\sigma_{h}$. Following the experimental indications of EMC and HERMES, we take $\sigma_{q}=0$ and $\sigma_{*}=\sigma_{h}=20$ mbarn for charged hadrons. With the latter assumption we need to consider effectively only the formation time $\tau_{F}$ for the pre-hadronic state [5]:

$$
\tau_{F}=\left(\frac{-\ln \left(z^{2}\right)-1+z^{2}}{1-z^{2}}\right) \frac{z \nu}{\kappa}
$$

where $\kappa$ is a parameter which we fix to $\kappa=0.25 \mathrm{GeV} / \mathrm{fm}$. Note that $\tau_{F} \rightarrow \frac{1}{\kappa}(1-z) \nu$ as $z \rightarrow 1$, giving the formation time suggested by the gluon bremsstrahlung model of Ref. [6]. Finally, nuclear absorption effects are included in the computations by multiplying the integrand in (7) by the nuclear absorption factor $\mathcal{N}_{A}$, which represent the fraction of hadrons which escape from the nucleus:

$$
\mathcal{N}_{A}=\int d^{2} b \int_{-\infty}^{\infty} d y \rho_{A}(b, y)\left[S_{A}(b, y)\right]^{A-1},
$$

where $\rho_{A}(b, y)$ is the nuclear density normalized to 1 at transverse and longitudinal coordinates $(b, y)$, and $S_{A}(b, y)$ is the survival probability of a hadron produced in $(b, y)$ :

$$
S_{A}(b, y)=1-\sigma_{h} \int_{y}^{\infty} d y^{\prime} \rho_{A}\left(b, y^{\prime}\right)\left(1-e^{-\left(y^{\prime}-y\right) / \tau_{F}}\right) .
$$

For the deuteron we used as a density the sum of the Reid's soft-core S- and D-wave functions squared 114. For heavier nuclei we used a Woods-Saxon density with radius $R_{A}=1.12 A^{1 / 3}-0.86 A^{-1 / 3} \mathrm{fm}$.

The $\nu$ - and $z$-dependence of the multiplicity ratio at EMC and HERMES after the inclusion of nuclear absorption are shown in Fig. 2 $1 \mathrm{~b}$ and Fig. 2 $2 \mathrm{c}$. It is clearly seen that while the CJRR model gives a nice description of the data, the NP model is ruled out.

The average values of $\langle\nu\rangle$ available at HERMES are smaller than at EMC and the absorption effects larger. In Fig. 3 we compare the $z$-distributions in the partial deconfinement models at EMC and HERMES before and after inclusion of absorption. As 
expected, at EMC with a ${ }^{63} \mathrm{Cu}$ target nuclear absorption is marginal and rescaling alone gives a satisfactory description of the data. At HERMES with a ${ }^{84} \mathrm{Kr}$ target, which is comparable to copper, both effects are larger and absorption is dominant, tending to mask rescaling effects. With a ${ }^{14} \mathrm{~N}$ target, which is smaller than krypton, both effects are smaller.

In summary, rescaling models (supplemented by nuclear absorption) are shown to be able to describe both HERMES and EMC data on the nuclear modification of hadron production in DIS processes. While the maximal deconfinement model is ruled out by the data - it assumes a too large deconfinement - the partial deconfinement model is shown to be a good one. Further precise data at moderate and high $\nu$ 's and for light and heavy targets are needed to disentangle rescaling and formation time effects.

\section{Acknowledgments}

We are grateful to N. Bianchi, P. di Nezza, B. Kopeliovich and V. Muccifora for stimulating discussions and to R. Fabbri for technical support during the conference.

\section{REFERENCES}

1. L. S. Osborne et al., Phys. Rev. Lett. 40 (1978) 1624.

2. J. Ashman et al. [European Muon Collaboration], Z. Phys. C 52 (1991) 1.

3. A. Airapetian et al. [HERMES Collaboration], Eur. Phys. J. C 20 (2001) 479; V. Muccifora [HERMES Collaboration], arXiv:hep-ex/0106088.

4. A. Bialas and T. Chmaj, Phys. Lett. B 133 (1983) 241.

5. A. Bialas and M. Gyulassy, Nucl. Phys. B 291 (1987) 793.

6. B. Kopeliovich, J. Nemchik and E. Predazzi, arXiv:nucl-th/9607036, published in proceedings of Workshop on Future Physics at HERA, Hamburg, Germany, 30-31 May 1996.

7. X. F. Guo and X. N. Wang, Phys. Rev. Lett. 85 (2000) 3591; J. w. Qiu and G. Sterman, arXiv:hep-ph/0111002; E. Wang and X. N. Wang, arXiv:hep-ph/0202105.

8. V. Muccifora [HERMES Collaboration], these proceedings.

9. O. Nachtmann and H. J. Pirner, Z. Phys. C 21 (1984) 277.

10. R. L. Jaffe, F. E. Close, R. G. Roberts and G. G. Ross, Phys. Lett. B 134 (1984) 449; Phys. Rev. D 31 (1985) 1004.

11. J. Dias de Deus, Phys. Lett. B 166 (1986) 98.

12. M. Gluck, E. Reya and A. Vogt, Eur. Phys. J. C5 (1998) 461.

13. S. Kretzer, Phys. Rev. D 62 (2000) 054001.

14. R. V. Reid, Annals Phys. 50 (1968) 411. 